PROCEEDINGS OF THE

AMERICAN MATHEMATICAL SOCIETY

Volume 128, Number 12, Pages 3661-3669

S 0002-9939(00)05478-2

Article electronically published on June 21, 2000

\title{
ON A CHARACTERIZATION OF FINITE VECTOR BUNDLES AS VECTOR BUNDLES ADMITTING A FLAT CONNECTION WITH FINITE MONODROMY GROUP
}

\author{
INDRANIL BISWAS, YOGISH I. HOLLA, AND GEORG SCHUMACHER \\ (Communicated by Ronald A. Fintushel)
}

\begin{abstract}
We prove that a holomorphic vector bundle $E$ over a compact connected Kähler manifold admits a flat connection, with a finite group as its monodromy, if and only if there are two distinct polynomials $f$ and $g$, with nonnegative integral coefficients, such that the vector bundle $f(E)$ is isomorphic to $g(E)$. An analogous result is proved for vector bundles over connected smooth quasi-projective varieties, of arbitrary dimension, admitting a flat connection with finite monodromy group.

When the base space is a connected projective variety, or a connected smooth quasi-projective curve, the above characterization of vector bundles admitting a flat connection with finite monodromy group was established by M. V. Nori.
\end{abstract}

\section{INTRODUCTION}

A vector bundle over a connected projective variety $X$ is called finite if there are two distinct polynomials, say $f$ and $g$, with nonnegative integral coefficients, such that the vector bundle $f(E)$ is isomorphic to $g(E)$ No1.

In [No1] Nori proved that a rank $n$ vector bundle $E$ over $X$ is finite if and only if it is given by a representation of $\pi_{1}(X)$ into $G L(n, \mathbb{C})$ which factors through a finite group. Equivalently, $E$ is finite if and only if it admits a flat connection, compatible with its holomorphic structure, such that the monodromy group is finite. That a vector bundle which admits a flat connection with finite monodromy group is actually a finite vector bundle was established in [We.

In [No2], the above result of [No1] was extended to the context of a smooth quasi-projective curve.

Let $X$ be a compact connected Kähler manifold, and let $E$ be a holomorphic vector bundle over $X$. We prove that $E$ admits a flat connection compatible with the holomorphic structure and with finite monodromy group, if and only if there are two distinct polynomials, say $f$ and $g$, with nonnegative integral coefficients, such that the two holomorphic vector bundles, namely $f(E)$ and $g(E)$, are isomorphic [Theorem 2.3]. The proof of the theorem is based on some results of [DPS] and [Si].

Received by the editors May 5, 1998 and, in revised form, March 1, 1999.

1991 Mathematics Subject Classification. Primary 53C07, 14F05.

Key words and phrases. Numerically flat vector bundle, flat connection, monodromy.

(C)2000 American Mathematical Society 
In Section 3, the result of [No2] on connected smooth quasi-projective curves has been extended to the more general situation of connected smooth quasi-projective varieties of arbitrary dimension.

Given a connected smooth quasi-projective variety $X_{0}$ over $\mathbb{C}$, consider $X=$ $X_{0} \cup D$, where $X$ is a smooth projective variety and $D$ is a divisor on $X$ with normal crossings. Any flat connection $\nabla$ on $X_{0}$ gives rise to a parabolic vector bundle $E(\nabla)$ over $X$. The filtration over any component of $D$ defining the parabolic structure of $E(\nabla)$ is by locally free subsheaves. If the monodromy group of $\nabla$ is finite, then all the parabolic weights are rational numbers.

As before, define a parabolic vector bundle $E_{*}$ to be finite if there are two distinct polynomials $f$ and $g$ with nonnegative integral coefficients such that the parabolic vector bundle $f\left(E_{*}\right)$ is isomorphic to $g\left(E_{*}\right)$.

We prove that a parabolic vector bundle with rational parabolic weights and parabolic filtration by subbundles is finite if and only if it corresponds to a flat connection on $X_{0}$ with finite monodromy [Theorem 3.3].

\section{Finite VECtor BUNDLES OVER KÄHLER MANifOldS}

Let $X$ be a compact connected Kähler manifold. For a holomorphic vector bundle $E$ over $X$, and a polynomial $f(x)=\sum_{i=0}^{n} a_{i} x^{i}$, where $a_{i} \in \mathbb{N}$, define the vector bundle

$$
f(E) \quad:=\sum_{i=1}^{n} a_{i} E^{\otimes i}
$$

over $X$, where $a_{i} E^{\otimes i}$ denotes the direct sum of $a_{i}$ copies of $E^{\otimes i}$ and $E^{\otimes 0}$ denotes the trivial line bundle. Following [No1] we define

Definition 2.1. A holomorphic vector $E$ over $X$ is called finite if and only if there are two distinct polynomials, say $f$ and $g$, of the above type, such that the vector bundle $f(E)$ is isomorphic to $g(E)$.

We will recall a construction of finite vector bundles. Let $\rho$ denote the homomorphism from the fundamental group $\pi_{1}\left(X, x_{0}\right)$ to $G L(V)$, where $V$ is a finite dimensional complex vector space, given by a composition of homomorphisms

$$
\pi_{1}\left(X, x_{0}\right) \longrightarrow G \stackrel{\rho^{\prime}}{\longrightarrow} G L(V)
$$

where $G$ is a finite group. Let $E_{\rho}$ denote the flat vector bundle over $X$ corresponding to the representation $\rho$.

The following simple proposition gives examples of finite vector bundles.

Proposition 2.2. The above vector bundle $E_{\rho}$ is finite.

Proof. Since $G$ is a finite group, any complex $G$-module is completely reducible, and furthermore, there are only finitely many isomorphism classes of irreducible $G$-modules. Thus there are two distinct polynomials $f$ and $g$, with nonnegative integral coefficients, such that the two $G$-modules, namely $f(V)$ and $g(V)$, are isomorphic. This immediately implies that the vector bundle $f\left(E_{\rho}\right)$ is isomorphic to $g\left(E_{\rho}\right)$. In other words, $E_{\rho}$ is finite.

The following theorem shows that the converse of the above proposition is valid. 
Theorem 2.3. A holomorphic vector bundle over $X$ is finite if and only if it corresponds to a representation of a finite quotient of the fundamental group of $X$, or equivalently, if and only if it admits a flat connection, compatible with the holomorphic structure, and with finite monodromy group.

Proof. We will start by recalling some concepts introduced in Section 1 of DPS. A line bundle $L$ over a compact complex manifold $M$, equipped with a Hermitian metric $\omega$, is called nef if given any $\epsilon>0$, there is a smooth Hermitian metric $h_{\epsilon}$ on $L$ such that $\Theta\left(h_{\epsilon}\right)+\epsilon \omega$ is a nonnegative Hermitian form, where $\Theta\left(h_{\epsilon}\right)$ is the Chern curvature form. This definition actually does not depend on the choice of $\omega$. A vector bundle $V$ on $M$ is called nef if the tautological line bundle $\mathcal{O}_{\mathbb{P}(V)}(1)$ on $\mathbb{P}(V)$ is nef.

A vector bundle $V$ over $M$ is called numerically flat if both $V$ and $V^{*}$ are nef.

Lemma 2.4. If a holomorphic vector bundle $E$ over $X$ is finite, then $E$ is numerically flat.

Proof of Lemma 2.4. Since the Krull-Remak-Schmidt theorem is valid for coherent analytic sheaves on $X$ [At], a vector bundle $E$ is finite if and only if there are finitely many indecomposable vector bundles, say $V_{1}, V_{2}, \ldots, V_{m}$, on $X$ such that any tensor power $E^{\otimes i}$ has the following direct sum decomposition:

$$
E^{\otimes i}=\sum_{j=1}^{m} a_{i, j} V_{j},
$$

where each $a_{i, j}$ is a nonnegative integer.

Let $E$ be a finite vector bundle over $X$.

Fix Hermitian structures on each $V_{j}$. Using (2.5), this induces a Hermitian structure on each $E^{\otimes i}$. These Hermitian metrics on the tensor powers of $E$ clearly satisfy the curvature criterion in Theorem 1.12 (page 306) of [DPS]. Now Theorem 1.12 of [DPS] implies that $E$ is nef. The dual $E^{*}$ is also finite, as $E$ is finite. Thus $E$ must be numerically flat.

Theorem 1.18, page 311, of [DPS] says that a holomorphic vector bundle $E$ over $X$ is numerically flat if and only if it admits a filtration of vector subbundles of $E$

$$
E_{1} \subset E_{2} \subset \ldots \subset E_{i} \subset \ldots \subset E_{m-1} \subset E_{m}=E
$$

such that each quotient $E_{i+1} / E_{i}$ is given by a unitary representation of $\pi_{1}(X)$. In particular, each $E_{i+1} / E_{i}$ is a direct sum of stable vector bundles of vanishing Chern classes. Thus this theorem of [DPS] combines with Lemma 2.4 to give the following corollary:

Corollary 2.6. Let $E$ be a finite vector bundle over $X$. Then $E$ is semistable with $c_{j}(E)=0$ for all $j \geq 1$. Furthermore, $E$ admits a filtration

$$
0=E_{0} \subset E_{1} \subset E_{2} \subset \ldots \subset E_{j} \subset \ldots \subset E_{l-1} \subset E_{l}=E
$$

such that for all $i \in[1, l]$, the subsheaf $E_{i}$ is a subbundle of $E$ and $E_{i} / E_{i-1}$ is a stable vector bundle with $c_{j}\left(E_{i+1} / E_{i}\right)=0$ for all $j \geq 1$ and $i \geq 0$.

For a semistable Higgs bundle $E$ with $c_{1}(E)=0=c_{2}(E)$ and admitting a Higgs field preserving a filtration by subbundles of $E$ such that each subsequent quotient is a stable Higgs vector bundle of degree zero, in [Si, Lemma 3.5] Simpson has constructed a canonical flat connection on $E$. In this context we note that a 
theorem of K. Uhlenbeck and S.-T. Yau says that a polystable vector bundle $E$ over $X$, with $c_{1}(E)=0=c_{2}(E)$, admits a unique unitary flat connection UY].

This canonical flat connection has the following properties. The connection on a direct sum of Higgs bundles is the direct sum of the connections for the individual Higgs bundles. The same statement is valid for the tensor product. Also, if $E$ is the trivial vector bundle of arbitrary rank with zero Higgs field, then it gets the trivial connection. This construction in [Si] actually gives a bijective correspondence between isomorphism classes of flat connections and isomorphism classes of semistable Higgs bundles satisfying the condition $c_{1}(E)=0=c_{2}(E)$ and admitting a filtration, compatible with the Higgs field, such that each subsequent quotient is a stable Higgs vector bundle of degree zero.

Let $E$ be a finite vector bundle over $X$. Consider it as a Higgs bundle with zero Higgs field. Now Corollary 2.6 implies that this Higgs bundle satisfies all the conditions needed in Lemma 3.5 of Si] to have a canonical flat connection. Let $\nabla$ denote the canonical flat connection on $E$. We observe that since the Higgs field on $E$ has been set to be zero, the flat connection $\nabla$ is compatible with the holomorphic structure on $E$. In other words, flat sections for $E$ are holomorphic sections.

For a polynomial $h$ with nonnegative integral coefficients, let $h(\nabla)$ denote the connection on $h(E)$ induced by $\nabla$.

If $f(E)$ is isomorphic to $g(E)$, then from the above properties of the canonical connection in $[\mathrm{Si}]$ we conclude that the two flat connections, namely $f(\nabla)$ and $g(\nabla)$, are isomorphic.

Fix two polynomials

$$
f(x)=\sum_{i=0}^{n+1} a_{i} x^{i} \quad \text { and } \quad g(x)=\sum_{i=0}^{n} b_{i} x^{i},
$$

where $a_{i}, b_{i} \in \mathbb{N}$ and $a_{n+1} \neq 0$, such that the vector bundle $f(E)$ is isomorphic to $g(E)$.

Let

$$
\rho: \pi_{1}\left(X, x_{0}\right) \longrightarrow G L(V)
$$

be the monodromy representation corresponding to the connection $\nabla$, where $V$ is the fiber $E_{x_{0}}$ of $E$ at $x_{0}$. The group $\pi_{1}\left(X, x_{0}\right)$ acts on the tensor algebra $\otimes V$ using $\rho$. An isomorphism between the two flat connections $f(\nabla)$ and $g(\nabla)$ induces an isomorphism, as $\pi_{1}\left(X, x_{0}\right)$-modules, between $f(V)$ and $g(V)$.

The proof of the theorem will be completed once we are able to establish the following proposition.

Proposition 2.8. Let $G$ be a finitely generated subgroup of $G L(V)$ such that $f(V)$ is isomorphic to $g(V)$ as $G$-modules, where $f$ and $g$ are two distinct polynomials as in (2.7). Then $G$ must be a finite group.

Setting $G$ to be the image $\rho\left(\pi_{1}\left(X, x_{0}\right)\right)$, Proposition 2.8 states that the monodromy of the connection $\nabla$ is a finite group.

Proof of Proposition 2.8. Any finitely generated subgroup of $G L(n, \mathbb{C})$ contains a subgroup of finite index which is torsion-free [Ra, Theorem 6.11, page 93]. Consequently, it suffices to show that every element of $G$ is torsion.

We will first show that all the eigenvalues of any element of $G$ are torsion.

Lemma 2.9. Take any $\gamma \in G$. All the eigenvalues of $\gamma$ are roots of unity. 
Proof of Lemma 2.9. From the given condition that $f(V)$ and $g(V)$ are isomorphic as $G$-modules it is easy to deduce that there are finitely many $G$-modules, say $V_{1}, V_{2}, \ldots, V_{k}$, such that every $\bigotimes^{j} V$ admits a decomposition $\sum_{i=1}^{k} a_{j, i} V_{i}$ as $G$ modules, where $a_{j, i}$ are nonnegative integers. So there are finitely many complex numbers, say $\left\{\lambda_{i}\right\}_{1 \leq i \leq N}$, such that all the eigenvalues for the action of $\gamma$ on any $\otimes^{j} V$ are contained in $\left\{\lambda_{i}\right\}_{1 \leq i \leq N}$.

Since the $i$-th power of an eigenvalue for the action of $\gamma$ on $V$ becomes an eigenvalue for the action of $\gamma$ on $\bigotimes^{i} V$, from the above remark it is immediate that all the eigenvalues for the action of $\gamma$ on $V$ must be roots of unity. This completes the proof of the lemma.

Now the proof of the proposition is completed by the following lemma.

Lemma 2.10. If $g \in G$ is a matrix with 1 as its only eigenvalue, then the equality $g=I d_{V}$ is valid.

Proof of Lemma 2.10. Let $A_{n}$ denote the $(n+1) \times(n+1)$ matrix whose $(i, j)$-th entry is 1 if $i=j$ or $i+1=j$ and 0 otherwise. Then $A_{n}$ has a standard action on $\mathbb{C}^{n+1}$ which is indecomposable. We will denote $\mathbb{C}^{n+1}$ equipped with the action of $A_{n}$ by $V_{n}$.

The proof of the lemma will be completed once we are able to establish the following assertion:

If $n \geq m$, then the Jordan canonical form of $A_{n} \otimes A_{m}$ has the form

$$
A_{n+m} \oplus A_{n+m-2} \oplus \ldots \oplus A_{n-m} .
$$

That the assertion indeed implies the lemma can be seen in the following way.

The indecomposable components which occur in $V$ for the action of $g$ are of the form $V_{n}$ for some $n$. If $g \neq I d_{V}$, then not all such $n$ are zero. Since there are only finitely many indecomposable components occurring in all tensor powers of $V$, we conclude that there will only be finitely many indecomposable components occurring in all tensor powers of $V_{n}$ for the action of $A_{n}$. On the other hand, the assertion implies that $V_{k n}$ occurs as an indecomposable component for the action of $A_{n}^{\otimes k}$ on $\bigotimes^{k} V_{n}$. Hence $V_{k n}$ occurs as an indecomposable component of $\bigotimes^{k} V$ for all $k$, which is a contradiction.

Now, to prove the assertion, let $E$ denote the standard two dimensional representation of $S L(2, \mathbb{C})$, and let $\operatorname{Sym}^{n} E$ be its $n$-th symmetric power. Denote by $A$ the element

$$
A:=\left(\begin{array}{ll}
1 & 1 \\
0 & 1
\end{array}\right)
$$

of $S L(2, \mathbb{C})$. For the representation $\operatorname{Sym}^{n} E$ of $S L(2, \mathbb{C})$, there exists a basis with respect to which $A$ acts by the matrix $A_{n}$. The assertion now follows from the fact that for $n \geq m$, the tensor product $\operatorname{Sym}^{n} E \otimes \operatorname{Sym}^{m} E$ is equivalent to

$$
\operatorname{Sym}^{n+m} E \oplus \operatorname{Sym}^{n+m-2} E \oplus \ldots \oplus \operatorname{Sym}^{n-m+2} E \oplus \operatorname{Sym}^{n-m} E
$$

as $S L(2, \mathbb{C})$ modules $([\mathrm{FH}]$, page 151, Ex. 11.11). This completes the proof of the lemma.

We already noted that Lemma 2.10 completes the proof of the assertion that any finite vector bundle over $X$ is given by a representation of a finite quotient of the fundamental group of $X$. The converse to it was already observed in Proposition 2.2. This completes the proof of the theorem. 
Remark 2.11. A principal $G$-bundle $P$ over $X$, where $G$ is a reductive algebraic group over $\mathbb{C}$, is called finite if for every finite dimensional complex representation $\rho: G \longrightarrow \operatorname{Aut}(V)$, the associated vector bundle $P(\rho)$ is finite. It is enough to check the criterion for one faithful representation. For $\rho$, as above, $\operatorname{Aut}(V) / G$ is affine. So constant maps are the only holomorphic maps from $X$ to $\operatorname{Aut}(V) / G$. From this fact and Theorem 2.3 it is straight-forward to deduce that a principal $G$-bundle over $X$ is finite if and only if it admits a flat connection with finite monodromy group.

In the next section we will consider representations of finite quotients of the fundamental group of a connected smooth quasi-projective variety.

\section{Flat CONNECTIONS With Finite MONODROMY OVER CONNECTED SMOOTH QUASI-PROJECTIVE VARIETIES}

Let $X$ be a connected smooth projective variety over $\mathbb{C}$. Let $D$ be a normal crossing divisor on $X$. By this we mean that $D$ is a reduced effective divisor such that each irreducible component of $D$ is smooth, and furthermore, the irreducible components intersect transversally. The complement $X-D$ will be denoted by $X_{0}$.

Let

$$
\rho: \pi_{1}\left(X_{0}\right) \longrightarrow U(n)
$$

be a unitary representation. The corresponding unitary flat bundle over $X_{0}$ will be denoted by $E(\rho)$. This flat connection, in general, does not extend across $D$. However, there is a natural extension of $E(\rho)$ as a holomorphic vector bundle over $X[\mathrm{De}]$. Denoting this vector bundle over $X$ by $\bar{E}$, the unitary flat connection on $E_{\rho}$ extends as a logarithmic singular connection on $\bar{E}$.

The vector bundle $\bar{E}$ has a natural parabolic structure over $D$ (the basic definitions of parabolic vector bundles can be found in $[\mathrm{MS}$, [MY]). The parabolic structure on $\bar{E}$ over an irreducible component $D_{i}$ of $D$ is determined by the residue of the logarithmic singular connection along $D_{i}$. A parabolic vector bundle arising this way has the property that the parabolic filtration over $D_{i}$ is actually a filtration of subbundles of $\left.\bar{E}\right|_{D_{i}}$. (In general a parabolic structure is given by a filtration of coherent subsheaves over $D_{i}[\mathrm{MY}$.)

If the monodromy of the unitary connection around $D_{i}$ is of finite order, then all the parabolic weights for the parabolic structure over $D_{i}$ are rational numbers.

For another unitary representation $\rho_{1}$, the parabolic vector bundle corresponding to the representation $\rho \oplus \rho_{1}$ is simply the direct sum of the corresponding parabolic vector bundles. The parabolic vector bundle corresponding to $\rho \otimes \rho_{1}$ is the parabolic tensor product of the corresponding parabolic vector bundles. (See [Bi1], Y] for the definition of the parabolic tensor product.)

For a parabolic vector bundle $E_{*}$ and a polynomial $g$ as in (2.7), define $g\left(E_{*}\right)$ to be the parabolic vector bundle constructed just as in Section 2 with the usual tensor product being replaced by the parabolic tensor product.

Since any finite subgroup of $G L(n, \mathbb{C})$ is conjugate to a subgroup of $U(n)$, any representation

$$
\rho^{\prime}: \pi_{1}\left(X_{0}\right) \longrightarrow G L(n, \mathbb{C})
$$


whose image is a finite subgroup of $G L(n, \mathbb{C})$ gives rise to a parabolic vector bundle over $X$ with parabolic structure over $D$ and satisfying the following two conditions:

1. all the parabolic weights are rational numbers;

2. the filtration over any irreducible component $D_{i}$, of $D$, defining the parabolic structure, is actually a filtration by subbundles.

Let $E_{*}$ denote this parabolic vector bundle corresponding to $\rho^{\prime}$.

Now, from the proof of Proposition 2.2 we conclude that there are two distinct polynomials, say $f$ and $g$, as in (2.7), such that the parabolic vector bundle $f\left(E_{*}\right)$ is isomorphic to $g\left(E_{*}\right)$.

The converse to this is also valid, as shown in the following theorem.

Theorem 3.3. Let $E_{*}$ be a parabolic vector bundle over $X$, with $E$ as the underlying vector bundle, and with a parabolic structure over $D$ which satisfies the following three conditions:

1. all the parabolic weights are rational numbers;

2. the parabolic filtration over any irreducible component $D_{i}$ of $D$ is defined by a filtration of subbundles of $\left.E\right|_{D_{i}}$;

3. there are two distinct polynomials $f$ and $g$, as in (2.7), such that the parabolic vector bundle $f\left(E_{*}\right)$ is isomorphic to $g\left(E_{*}\right)$.

Then there is a representation $\rho: \pi_{1}\left(X_{0}\right) \longrightarrow U(n)$, with $\rho\left(\pi_{1}\left(X_{0}\right)\right)$ a finite subgroup of $U(n)$, such that the parabolic vector bundle corresponding to $\rho$ is isomorphic to $E_{*}$. (The validity of the converse of the statement was observed earlier.)

Proof. Fix a positive integer $N$ such that all the parabolic weights of $E_{*}$ are of the form $m / N$, where $m$ is an integer.

Let

$$
D=\sum_{i=1}^{l} D_{i}
$$

be the decomposition of $D$ into its irreducible components.

The "Covering Lemma" of Y. Kawamata [KMM] Theorem 1.1.1] says that there are a connected projective manifold $Y$ and a Galois covering morphism

$$
p: Y \longrightarrow X
$$

such that the reduced divisor $\left(p^{*} D\right)_{\text {red }}$ is a normal crossing divisor on $Y$ and furthermore, $p^{*} D_{i}=k_{i} N$. $\left(p^{*} D_{i}\right)_{\text {red }}$, where $k_{i}, 1 \leq i \leq l$, are positive integers. Let $\Gamma$ denote the Galois group for the covering map $p$.

A construction of [Bi2] (we will not describe it here since it has already been repeated in [Bi1], $\mathrm{Bi3}$, $[\mathrm{BN}]$, etc.) gives a one-to-one correspondence between the collection of parabolic vector bundles over $X$, with parabolic structure over $D$ which satisfies the first two conditions in the statement of the theorem, and the collection of a certain class of $\Gamma$-linearized vector bundles over $Y$. The class of $\Gamma$-linearized vector bundles in question satisfy the condition that, for any point $y \in Y$, the action of the isotropy subgroup $\Gamma_{y} \subseteq \Gamma$ on the fiber of the vector bundle over $y$ is of order $N$, and the action of $\Gamma_{z}$, where $z \in Y-p^{-1}(D)$, is the trivial action.

Let $W$ denote the $\Gamma$-linearized vector bundle corresponding to the parabolic vector bundle $E_{*}$. The above mentioned construction of [Bi2] takes the parabolic tensor product (respectively, direct sum) of parabolic vector bundles to the usual tensor product (respectively, direct sum) of the corresponding $\Gamma$-linearized vector bundles 
equipped with the induced $\Gamma$-linearization. Thus the given condition $f\left(E_{*}\right)=g\left(E_{*}\right)$ implies that the following is valid:

$$
f(W)=g(W)
$$

as $\Gamma$-linearized vector bundles.

Theorem 2.3 says that $W$ corresponds to a unitary representation of $\pi_{1}(Y)$. (This representation is unique up to a conjugation.) Let $\nabla$ denote the unique unitary flat connection on $W$.

Let $p^{\prime}$ denote the restriction of $p$ to $p^{-1}\left(X-D^{\prime}\right)$, where $D^{\prime}$ is the divisor on $X$ over which $p$ is ramified. The direct image

$$
V:=p_{*}^{\prime}\left(\left.W\right|_{p^{-1}\left(X-D^{\prime}\right)}\right)
$$

has a unitary flat connection induced by $\nabla$; this flat connection on $V$ will be denoted by $\nabla^{\prime}$. The $\Gamma$-linearization of $W$ induces an action of $\Gamma$ on the vector bundle $V$, i.e., we have a homomorphism

$$
\gamma: \Gamma \longrightarrow \operatorname{Aut}(V) \text {. }
$$

This action of $\Gamma$ is compatible with the flat connection $\nabla^{\prime}$ on $V$ in the sense that any automorphism $\gamma(g)$ of $V$ preserves $\nabla^{\prime}$. So the invariant part $V^{\Gamma}$, for the action of $\Gamma$ on $V$, has a natural unitary flat connection, which will be denoted by $\nabla^{\Gamma}$.

For any $y \in p^{-1}\left(X_{0}\right)$ the isotropy subgroup $\Gamma_{y} \subseteq \Gamma$ acts trivially on the fiber $W_{y}$ Bi2]. Hence the flat vector bundle $V^{\Gamma}$ extends to $X_{0}$. Let $V_{0}$ denote the unitary flat vector bundle over $X_{0}$ obtained by extending $V^{\Gamma}$.

Let $E_{*}^{\prime}$ denote the parabolic bundle over $X$, with parabolic structure over $D$, corresponding to the unitary flat vector bundle $V_{0}$ over $X_{0}$. It is easy to see that, in the correspondence between parabolic vector bundles and $\Gamma$-linearized vector bundles constructed in [Bi2], the parabolic vector bundle $E_{*}^{\prime}$ is actually isomorphic to $E_{*}$. This completes the proof of the theorem.

\section{ACKNowledgments}

A key Lemma 2.4, which is a consequence of Theorem 1.12 of [DPS], was pointed out to us by Jean-Pierre Demailly. We are very grateful to him for his kind help. Thanks are also due to M.S. Raghunathan, S. Ramanan and C.T. Simpson for useful discussions. We wish to thank the referee for a very careful reading of the paper. The first-named author thanks the Philipps-Universität, Marburg, for its hospitality.

\section{REFERENCES}

[At] M.F. Atiyah : On the Krull-Schmidt theorem with application to sheaves. Bull. Soc. Math. Fr. 84 (1956), 307-317. MR 19:172b

[Bi1] I. Biswas : Parabolic ample bundles. Math. Ann. 307 (1997), 511-529. MR 98e:14041

[Bi2] I. Biswas: Parabolic bundles as orbifold bundles. Duke Math. Journal 88 (1997), 305-325. MR 98m:14045

[Bi3] I. Biswas : Chern classes for parabolic bundles. Jour. Math. Kyoto Univ. 37 (1997), 597-613. CMP 98:13

[BN] I. Biswas and D.S. Nagaraj : Parabolic ample bundles, II: Connectivity of zero locus of a class of sections. Topology 37 (1998), 781-789. MR 99e:14047

[De] P. Deligne : Equations Différentielles à Points Singuliers Réguliers. Lecture Notes in Math. 163, Springer-Verlag Berlin-Heidelberg-New York 1970. [MR 54:5232

[DPS] J.-P. Demailly, T. Peternell and M. Schneider : Compact complex manifolds with numerically effective tangent bundles. Jour. Alg. Geom. 3 (1994), 295-345. MR 95f:32037 
[FH] W. Fulton and J. Harris : Representation theory: a first course. Graduate Texts in Math. 129, Springer-Verlag Berlin-Heidelberg-New York 1991. MR 93a:20069

[KMM] Y. Kawamata, K. Matsuda and K. Matsuki : Introduction to the minimal model problem. Adv. Stu. Pure Math. 10 (1987), 283-360. MR 89e:14015

[MS] V. Mehta and C.S. Seshadri : Moduli of vector bundles on curves with parabolic structure. Math. Ann. 248 (1980), 205-239. MR 81i:14010

[MY] M. Maruyama and K. Yokogawa : Moduli of parabolic stable sheaves. Math. Ann. 293 (1992), 77-99. MR 93d:14022

[No1] M.V. Nori : On the representations of the fundamental group. Compositio Math. 33 (1976), 29-41. MR 54:5237

[No2] M.V. Nori : The fundamental group scheme. Proc. Indian Acad. Sci. (Math. Sci.) 91 (1982), 73-122. MR 85g:14019

[Ra] M.S. Raghunathan : Discrete subgroups of Lie groups. Ergeb. Math. Grenz. Band 68, Springer-Verlag Berlin-Heidelberg-New York 1972. MR 58:22394a

[Si] C.T. Simpson : Higgs bundles and local systems. Pub. Math. I.H.E.S. 75 (1992), 5-95. MR 94d:32027

[UY] K. Uhlenbeck and S.-T. Yau : On the existence of Hermitian-Yang-Mills connections in stable vector bundles. Comm. Pure Appl. Math. 39 (1986), 257-293. MR 88i:58154

[We] A. Weil : Généralisation des fonctions abélinnes. Jour. Math. Pures et Appl. 17 (1938), $47-87$.

[Y] K. Yokogawa : Infinitesimal deformation of parabolic Higgs sheaves. Int. Jour. Math. 6 (1995), 125-148. MR 95k:14029

School of Mathematics, Tata Institute of Fundamental Research, Homi Bhabha ROAD, BOMBAY 400005, INDIA

E-mail address: indranil@math.tifr.res.in

School of Mathematics, Tata Institute of Fundamental Research, Homi Bhabha RoAD, BOMBAY 400005, INDIA

E-mail address: yogi@math.tifr.res.in

Fachbereich Mathematik der Philipps-Universität, Hans-Meerwein-Strasse, LahnBerge, D-35032 Marburg, Germany

E-mail address: schumac@mathematik.uni-marburg.de 